\title{
Bioanalysis
}

\section{Microspray and microflow LC-MS/MS: the perfect fit for bioanalysis}

\author{
"Looking to future, organizations will be looking for high-performance solutions \\ that improve productivity and efficiency while maintaining workflows."
}

Keywords: bioanalysis $\bullet$ LC-MS/MS $\bullet$ microflow $\bullet$ microspray $\bullet$ nanospray

The advantages of microflow LC (MFLC) and nanoflow liquid chromatography coupled with a mass spectrometer are well known and include improved signal, low solvent consumption, reduced matrix effects and reduced source contamination [1]. In the past, microflow and nanoflow MS/MS have mainly been used in academic and fundamental research labs requiring high sensitivity analysis with limited sample volumes for qualitative or comparative analysis. The analytical advantages of low flow methods have led the technological development of specialized LC pumps that accurately deliver $\leq 100 \mu \mathrm{l} / \mathrm{min}$ of solvent, for use with columns with an inside diameter of $\leq 1 \mathrm{~mm}$. With the use of these specialized LC pumps and rugged API-MS ionization sources, the realm of using MFLC-MS/MS for quantitative bioanalysis is a possibility.

Researchers have used nanoflow or MFLC-MS/MS for analysis of proteomic samples, food samples, the petroleum products and other applications [2,3]. Nanoflow LC-MS/MS publications as early as 1994 include applications in discovery bioanalysis [4]. However, application of nano or MFLC-MS/MS methods in regulated bioanalysis including application in pharmaceutical and clinical space has not been extensively studied. The adoption of MFLC-MS/ MS in regulated bioanalytical laboratories has been limited by the perception that the technique lacks sufficient robustness, and the technical hurdles of implementation. The high volume requirement of bioanalytical quantitation typically demands operation on a continuous 24-7 basis. Downtime is not acceptable. Fortunately, development on the instrumentation side from vendors, and the application side of leading bioanalytical labs, have fostered many developments in the design and coupling of sources for nanospray ESI-MS to improve the ruggedness and ease of use of MFLC-MS/MS. More recently a design successful in coupling the HPLC column within the conventional ESI has been shown to provide better signal and better chromatography compared with traditional segregated techniques [5].

\section{Integration of miniaturized LC with nanospray ESI-MS is a key for success}

LC-MS has experienced significant technical evolution having established trends toward decreasing column diameter, lower flow rates and smaller column packing particle sizes. State of the art 'conventional' LC has evolved from $4.6 \mathrm{~mm}$ inside diameter columns (ID), operating at ca. $1 \mathrm{ml} / \mathrm{min}$, to $1-2 \mathrm{~mm}$ ID columns, operating at less than $200 \mu \mathrm{l} / \mathrm{min}$. Even smaller micro- $(0.2-0.3 \mathrm{~mm}$ ID) and nanoscale $(<0.2 \mathrm{~mm}$ ID) column formats, operating at 10 and $0.3 \mu \mathrm{l} / \mathrm{min}$, respectively, have strong application specific roles, particularly when high sensitivity is required and/or sample volumes are strictly limited.

The driving factors in the trend to smaller diameter columns are many. Primary benefits in the switch to smaller columns include reduced solvent consumption, improved cleanliness of the mass spectrometer source/ inlet/vacuum system, and perhaps most importantly, reduced sample injection volume. A $0.3 \mathrm{~mm}$ ID column, on average,

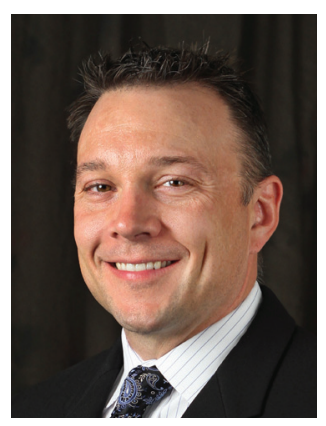

Shane R Needham Author for correspondence: Alturas Analytics, Inc., 1324 Alturas Drive, Moscow, ID 83843, USA sneedham@alturasanalytics.com

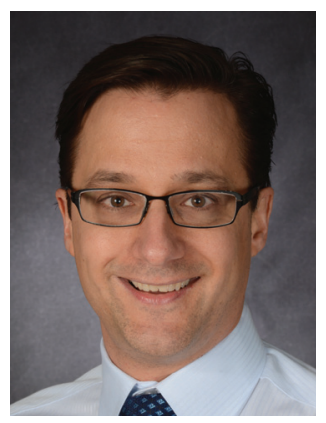

Gary A Valaskovic New Objective, Inc., 2 Constitution Way, Woburn, MA 01801, USA 
will consume one twentieth and one one-hundredth of the solvent required for 2 and $4.6 \mathrm{~mm}$ ID columns, respectively [6]. Less solvent consumption reduces both purchase and chemical waste disposal costs. The low flow rates associated with small ID columns achieve the same front-end cleanliness goals as the typical postcolumn LC divert valve approach. Less mobile phase flowing through the ESI source, and presented to the front-end inlet of the mass spectrometer, translates directly into a cleaner MS, reducing maintenance expense and increasing MS acquisition time without the need for a switching valve.

The reduced injection volume required by small ID columns, and the resulting relative concentration advantage when using reverse-phase gradient elution HPLC, results in clear experimental advantages. As shown previously, the concentration of analyte on the head of the column, for injections of a fixed volume, increases as column diameter decreases [7]. A $0.3 \mathrm{~mm}$ ID column has a 40 -fold concentration advantage compared with a $2.1 \mathrm{~mm}$ ID column, while a $75 \mu \mathrm{m}$ ID column has a nearly 800 -fold advantage [7]. This concentration benefit is often described in the literature as an 'increase in (column) sensitivity', it is, and however, perhaps more correctly viewed as an effective sampling advantage. Micro- and nanobore columns enable the handling and analysis of much smaller absolute sample sizes and/or concentration of trace components present in larger sample volumes. Reduced sampling volume has an under-appreciated workflow benefit, unrelated to sensitivity, giving incredible sample security or a 'peace of mind' to the analyst for unattended analyses. A precious $100 \mu \mathrm{l}$ sample is sufficient for at least 50 injections of $1.0 \mu \mathrm{l}$ with micro-scale LC, compared with only two $40 \mu \mathrm{l}$ injections using conventional LC. The reasons to implement $0.3 \mathrm{~mm}$ ID (and smaller) columns are as compelling as the initial push to switch to $2.1 \mathrm{~mm}$ ID columns. Certainly, it is hard to see those labs ever moving back to the routine use of $4.6 \mathrm{~mm}$ columns.

Along with the compelling advantages provided by miniaturized sampling and separation, detection by ESI-MS must also fit the paradigm of miniaturization. The driving factor to trend to lower flow rates for ESI-MS is an improvement in ionization efficiency. As shown by Kebarle et al. [8], early works on ESI response demonstrated an increase in proportional MS signal-to-noise ratio, as the mobile phase flow rate of ESI is reduced. Thus a system that provides optimal chromatographic benefits and optimal flow rates to the MS is an ideal situation for high-throughput, highsensitivity LC-MS bioanalysis. Nanobore chromatography, using LC columns having a typical ID of 300 $\mu \mathrm{m}$ or less, is conveniently coupled to MS via high flow nanospray ('microspray') ionization, a low-flow variant of ESI. Microspray provides a highly efficient means to transport liquid ions to gas phase ions with little, or no added thermal energy. The low-flow rates associated with nanospray $(10-500 \mathrm{nl} / \mathrm{min})$ result in the generation of submicrometer droplets, generating a maximal surface area-to-volume ratio of column effluent. Such high surface area translates directly to high ionization efficiency [9]. The lower thermal requirement for microspray compared with traditional ESI is an added benefit as it is directly compatible with high molecular weight, thermally sensitive, peptides, proteins and small molecules. Additional advantages at low-flow rates $(<100 \mu \mathrm{l} / \mathrm{min})$ include reduced ion suppression [10], a trend toward equimolar response [11], linear ionization response [12] and improved signal-to-noise ratio [13].

In addition to the smaller droplets afforded by lowflow rates, the overall physical size of the ESI plume also scales with flow rate. Conventional flow $(\mathrm{ml} / \mathrm{min})$ ESI-MS ESI generates an aerosol plume centimeters in diameter. Compared with the (sub) millimeter MS inlet, only a small fraction of the plume is sampled. Under conventional conditions, the MS inlet is an atmospheric pressure flow splitter, with greater than $99 \%$ of the generated plume flowing to waste. Highflow nanospray and microspray, with a (sub) $\mu \mathrm{l} / \mathrm{min}$ flow rate, has a plume that is on the same dimensional scale as the MS inlet [14]. This gain in apparent sensitivity is best thought of in terms of utilization and sampling efficiency. Near zero postcolumn waste directly translates into the ability to handle micro-scale samples with complete efficiency.

\section{Micro- \& nano-LC are well suited for quantitative bioanalysis}

It would seem that the rational combination of microor nano-LC combined with nanospray ionization, would yield a nearly ideal platform for high sensitivity bioanalysis by LC-MS/MS. In practiced hands, that is often the case, but experimental challenges are present. Assembling a high-performance system is at present time the domain of the expert user. Challenges in preand postcolumn plumbing, sample preparation of complex matrices, and mass spectrometer tuning requires training and persistence in method development.

A common misconception is that miniaturized LC is 'too slow' to use in applications requiring short run times and high throughput. The casual observation of the mobile phase flow rate $(0.3-10 \mu \mathrm{l} / \mathrm{min})$ would suggest this is the case. However, it is important to realize that the linear velocity of analyte through a micro- or nanobore column is identical to a conventional column. This misperception appears to 
have two different origins: many minutes (ten or more) were required to move mobile phase from the piston of the pump through the autosampler and onto the column. Second, much of the application literature for miniaturized LC is qualitative peptide analysis using injection-to-injection cycle times measured in hours or days. The reason for these long cycle times is not the speed of the sample flow through the column. The long cycle time is a necessity based on the mixture complexity faced in qualitative discovery experiments. Shallow gradients and long LC $(\geq 25 \mathrm{~cm})$ columns must be employed to achieve sufficient chromatographic peak capacity for the mass spectrometer to effectively sample the mixture.

Attention to the overall plumbing and connection scheme, starting at the outlet of the HPLC pump is required critical factors including total system volume, the injection volume, as well as pre- and postcolumn volume. Precolumn volume is clearly important as the gradient delay time from pump, through the autosampler, and onto the column, places the ultimate limit on the minimum injection cycle time. Controlling and reducing postcolumn volume is perhaps the most critical parameter to maintaining the quality of LC peak shape and overall cycle times. Integration of

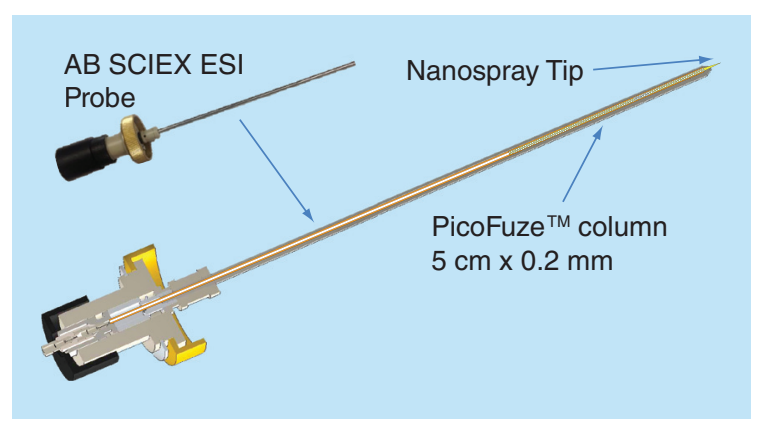

Figure 1. A PicoFuze ${ }^{T M}$ Column Integrated into an AB SCIEX ESI Probe.

the LC column into body the ionization emitter provides just such a benefit that will minimize connections and thus precolumn and postcolumn volumes. Indeed, the technology of the so-called 'packed-emitter' column (PicoFrit ${ }^{\mathrm{TM}}$ column,) has been the de facto standard in qualitative proteomics for the past 20 years [15]. More recently, integrated columns for use in microspray have shown the ruggedness and ease of use to have great utility in quantitative bioanalysis $[5,16]$. An LC column integrated into the ESI-MS source is shown in Figure 1 with a corresponding chromatogram shown in Figure 2. This integrated system is known as the PicoFuze ${ }^{\mathrm{TM}}$. As presented, the minimization of

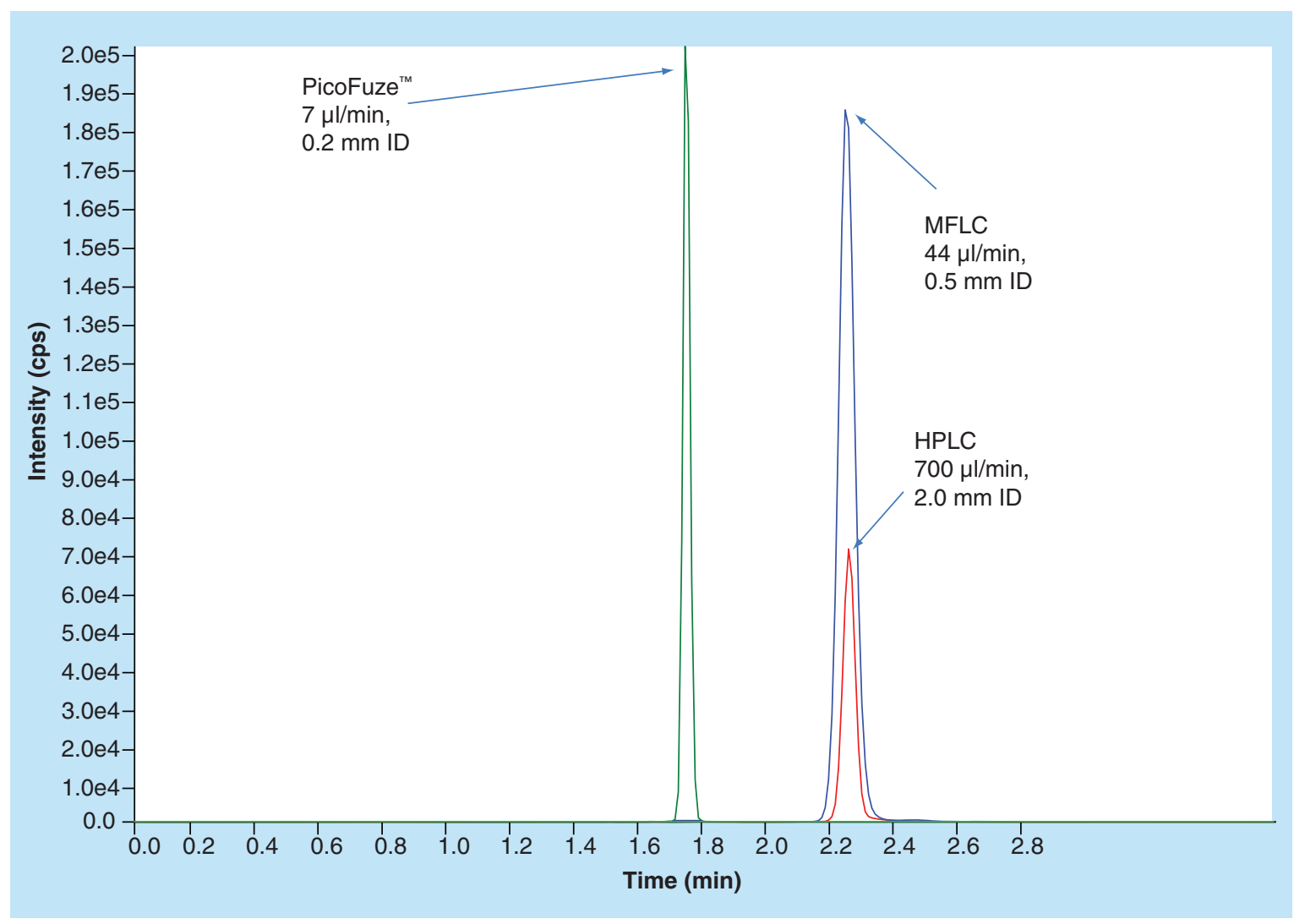

Figure 2. Overlayed Chromatograms from the PicoFuze ${ }^{T M}$, MFLC-MS/MS, and HPLC-MS/MS Analysis of MAOB Digest from Plasma. 
extra pre and postcolumn connection volumes yields improved chromatographic peak shape and signal to noise compared with nonintegrated LC columns. Additionally, unlike operation at conventional flow rates, no switching valve or external column heater is needed for these high performance experiments.

\section{Conclusion}

Looking to future, organizations will be looking for high-performance solutions that improve productivity and efficiency while maintaining workflows. Microflow-LC-MS, especially integrated column LC-MS are solutions that meet this need to support therapeutic advancements and clinical applications. For integrated systems, the reduction of solvent use, minimized source contamination, lower injection volumes, no need for switching valves or external column heaters, improves productivity and lower costs. The end result is an easy to use system that is also high performance. As in

\section{References}

1 Gibson GT, Mugo SM, Oleschuk RD. Nanoelectrospray emitters: trends and perspective. Mass Spectrom. Rev. 28(6), 918-936 (2009).

2 Wood TD, Moy MA, Dolan AR et al. Miniaturization of electrospray ionization mass spectrometry. Appl. Spec. Rev. 38(2), 187-244 (2003).

3 Wickremsinhe ER, Singh G, Ackermann BL, Gillespie TA, Chaudhary AK. A review of nanoelectrospray ionization applications for drug metabolism and pharmacokinetics. Curr. Drug Metab. 7(8), 913-928 (2006).

4 Skor H, Visswanathan R. Quantitative bioanalysis by microflow LC-MS to support discovery-based pharmacokinetic studies. In: Eliminating Bottlenecks for Efficient Bioanalysis: Practices and Applications in Drug Discovery and Development. Shou WZ, Weng N (Eds). Future Science Ltd, London, UK 66-84 (2014).

5 Johnson C, Christianson C, DeChenne S, Needham S, Valaskovic G. Validation of an in-source micro flow LC-MS/ MS method for bioanalysis. Proceedings from the 62nd Conference on American Society for Mass Spectrometry. Baltimore, MD (2014). www.asms.org

6 Chapter 4 HPLC Columns for Pharmaceutical Analysis. In: Handbook of Pharmaceutical Analysis by HPLC. Ahuha S, Dong M (Eds). Elsevier Hobooken, NJ 91 (2005).

7 Tomer KB, Moseley MA, Deterding LJ, Parker CE. Capillary liquid chromatography/mass spectrometry. Mass Spectrom. Rev. 13, 431-457 (1994).

8 Kebarle P, Tang L. From ions in solution to ions in the gas phase: the mechanism of electrospray ionization mass spectrometry. Anal. Chem. 65, 972A-986A (1993). many technology-driven industries, the miniaturization of process, components and workflow provides optimal results - we see this to be no different for the future of LC-MS.

\section{Acknowledgements}

The authors wish to thank M Lee of Milestone Development Services for insightful discussions during the preparation of this manuscript.

\section{Financial \& competing interests disclosure}

The authors have no relevant affiliations or financial involvement with any organization or entity with a financial interest in or financial conflict with the subject matter or materials discussed in the manuscript. This includes employment, consultancies, honoraria, stock ownership or options, expert testimony, grants or patents received or pending, or royalties.

No writing assistance was utilized in the production of this manuscript.

9 Juraschek R, Dulcks T, Karas M. Nanoelectrospray-more than just a minimized-flow electrospray ionization source. J. Am. Soc. Mass Spectrom. 10, 300-308 (1999).

10 Hatsis P, Valaskovic G, Wu JT. Online nanoelectrospray/ high-field asymmetric waveform ion mobility spectrometry as a potential tool for discovery pharmaceutical bioanalysis. Rapid Commun. Mass Spectrom. 23(23), 3736-3742 (2009).

11 Valaskovic GA, Utley L, Lee MS, Wu JT. Ultra-low flow nanospray for the normalization of conventional liquid chromatography/mass spectrometry through equimolar response: standard-free quantitative estimation of metabolite levels in drug discovery. Rapid Commun. Mass Spectrom. 20 (7), 1087-1096 (2006).

12 Wickremsinhe ER, Singh G, Ackermann BL, Gillespie TA, Chaudhary AK. A review of nanoelectrospray ionization applications for drug metabolism and pharmacokinetics. Curr. Drug Metab. 7(8), 913-928 (2006).

13 Zhou F, Lu Y, Ficarro SB, Webber JT, Marto JA. Nanoflow low pressure high peak capacity single dimension LC-MS/ MS platform for high-throughput, in-depth analysis of mammalian proteomes. Anal. Chem. 84(11), 5133-5139 (2012)

14 Schneider BB, Javaheri H, Covey TR. Ion sampling effects under conditions of total solvent consumption. Rapid Commun. Mass Spectrom. 20(10), 1538-1544 (2006).

15 Emmett MR, Caprioli RM. Micro-electrospray mass spectrometry: ultra-high-sensitivity analysis of peptides and proteins. J. Am. Soc. Mass Spectrom. 5(7), 605-613 (1994).

16 Gallagher R, Dillon L, Grimsley A, Murphy J, Samuelsson $\mathrm{K}$, Douce D. The application of a new microfluidic device for the simultaneous identification and quantitation of midazolam metabolites obtained from a single micro-litre of chimeric mice blood. Rapid Commun. Mass Spectrom. 28(11), 1293-1302 (2014). 